\title{
Development of Nanoporous Gold- Based Supercapacitor
}

\author{
Ji Li ${ }^{a}$, Jinshan $\mathrm{Yu}^{\mathrm{b}}$ \\ National University of Defense and Technology, Changsha 410000, China \\ aljnjuceas@163.com, bjjss_y@163.com
}

Keywords: Electrochemical capacitor, Nanoporous gold, Double-layer capacitor.

\begin{abstract}
In light of excellent power density and extended cycling stability, supercapacitors have been recognized as the advanced energy storage in the future. Nanoporous gold with a large internal surface and excellent conductivity, is a promising electrode material for supercapacitors. This article reviews latest progress in capacitive application of nanoporous gold and nanoporous gold-based composite.
\end{abstract}

\section{Introduction}

Owing to general concerns about limited oil storage and global warming situation, there is growing demand for alternative green energy storage and conversion systems including batteries, fuel cells, and supercapacitors [1-4]. Electrochemical supercapacitors(SCs) have attracted particular attention in light of their excellent power density and extended cycling stability[5]. Typically the SCs exhibit 20-200times larger capacitance per unit volume or mass than conventional capacitors [6]. In general, supercapacitor system contains electrode, electrolyte and separator. Numerous efforts have been made to develop advanced electrode materials for high-performance SCs by making use of surface ion adsorption, referred as double-layer capacitance, and surface redox reactions, referred as pseudocapacitance [7].

Nanoporous gold is a corrosion-derived nanostructured material. It is generated by the corrosion of an alloy comprised of $\mathrm{Au}$ and a less noble metal, such as $\mathrm{Ag}, \mathrm{Cu}$ or $\mathrm{Al}$. The electrochemical removal (dealloying) of the less noble constituent results in an open bicontinuous nanoporous network of interconnected ligaments comprised almost entirely gold[8]. The difference in the compositions of the precursor alloys, the dealloying conditions and subsequent thermal annealing of dealloyed materials will result in remarkble dissimilarity of pore sizes, which may effect the capacitive property of electrode. Furthermore, the plastic deformation of nanoporous gold under compressive stress was studied by depth-sensing nanoindentation[9]. NPG with a relative density of $42 \%$ exhibit a mean hardness of $145( \pm 11) \mathrm{MPa}$ and aYoung's modulus of $11.1( \pm 0.9) \mathrm{Gpa}$, potentially opening a door to a class of high yield strength—low-density materials,such as supercapacitor electrodes.

\section{NPG-based supercapacitors.}

\subsection{NPG for electric double-layer capacitors (EDLCs)}

Electric double-layer capacitors are based on the accumulation of ions of opposite charges in a double layer at electrodes. The charging and discharging process does not give rise to any chemical phase and composition change, and thus the EDLCs have relatively much better cyclability. In recent years, carbon material such as graphene and carbon nanotubes as well as activated cabon has been widely reported as electrode for EDLCs [10]. On the other hand, porous metals with a large internal surface and excellent conductivity are also promising electrode for supercapacitors, which have not been well investigated.

X.Y. Lang et al. fabricated nanoporous gold(NPG) sheets with a thickness of $30 \mu \mathrm{m}$ by delloying $\mathrm{Ag}_{65} \mathrm{Au}_{35}$ (at.\%) ribbons(as shown in Fig.1[11]). The dealloying process was performed by free corrosion in a $70 \% \mathrm{HNO}_{3}$ solution for $24 \mathrm{~h}$ at room temperature, followed by pure water quenching. The dealloyed samples have quasi-periodic nanoporous channels and gold ligaments with average size of $\sim 40 \mathrm{~nm}$. After synthesizing NPG sheets into SC device, electrochemical experiments were 
carried out using a classic two-electrode configuration in a $2 \mathrm{M} \mathrm{KOH}$ aqueous solution and a N,Ndiethyl-N-methyl-N-(2-methoxyethyl)ammonium bis(trifluoromethanesulfonyl) imide (DEMETFSI) room temperature ionic liquid (RTIL), repectively. The calculated energy density of the SC device was determined to be $16.5-21.7 \mathrm{mWh} \mathrm{cm}^{-3}$, which is 2-4 times higher than those with the $\mathrm{KOH}$ solution $\left(4.89-8.31 \mathrm{mWh} \mathrm{cm}^{-3}\right)$, mainly because of the higher operation voltage given by low ionic mobility of the ionic liquid.

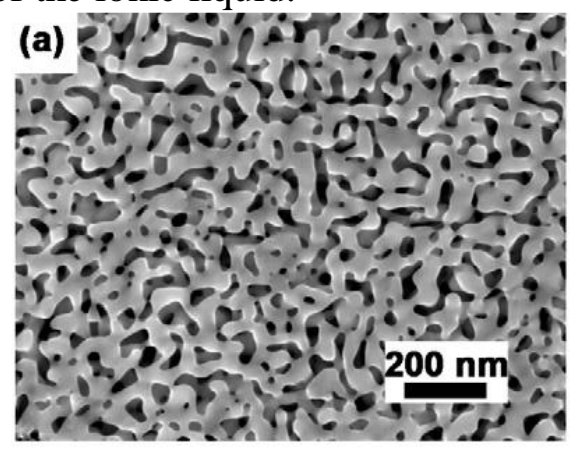

(c)

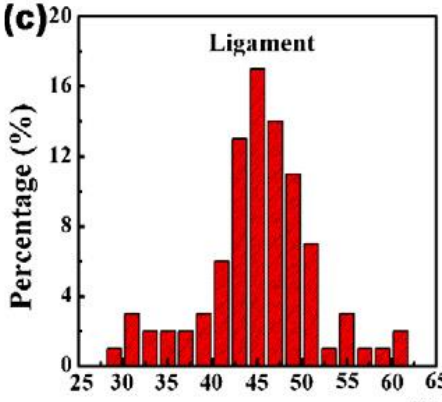

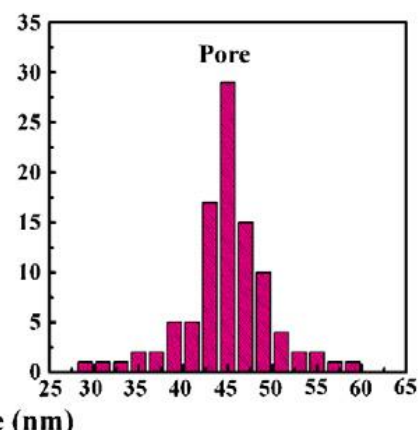

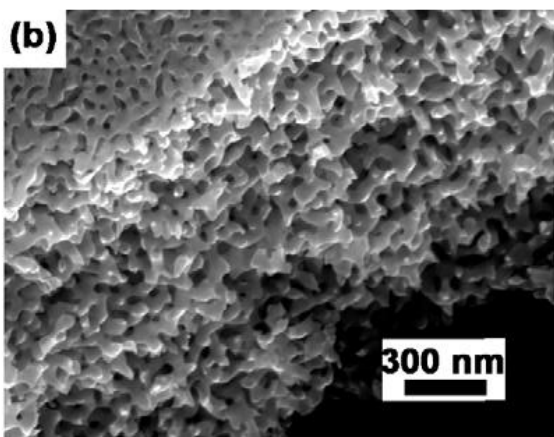

(d) Conductive wire

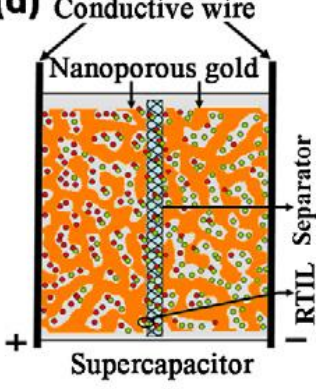

Fig. 1 Representative (a) top view and (b) cross-section SEM images of a NPG electrode. (c) Size distributions of gold ligaments and nanoporous channels of the NPG sample. (d) Schematic diagram of the supercapacitor assembled with NPG as both electrodes and current collectors, and a piece of cotton paper as separator [11]

\subsection{NPG for pesudocapacitors}

To overcome the low energy density of electrochemical double-layer capacitors, great efforts have been devoted to studying transition metal oxides $\left(\mathrm{RuO}_{2}\right.$ [12], $\mathrm{MnO}_{2}$ [13], $\mathrm{NiO}$ [14], $\mathrm{CoO}_{\mathrm{x}}$ [15]) and conductive polymers (PPy, PANI) which exhibit typical pseudocapacitance. Thus make the pesudocapacitors display much higher specific capacitance than double-layer capacitors, where the mechanism of fast and Faradic surface redox reaction offers the high energy storage. On the other hand,the poor conductivity and anomalous structure essentially limits the practical uses of pseudocapacitors. Accordingly, nanoporous gold with bicontinuous structure, uniform pore size and excellent conductivity is a promising substrate and current collector for electrode-active materials.

$\mathrm{MnO}_{2}$ has attracted intense attention because of its low cost, environmental friendliness, and high theoretical capacitance $\left(1,370 \mathrm{~F} \cdot \mathrm{g}^{-1}\right)$. Designing composite materials by depositing $\mathrm{MnO}_{2}$ onto a conductive substance is a feasible strategy to address the poor electrical conductivity of $\mathrm{MnO}_{2}$. Henghui Xu et al. reported on fabrication of NPG@ $\mathrm{MnO}_{2}$ composite electrode (as shown in Fig.2 [16]). The electrodeposition of $\mathrm{MnO}_{2}$ was carried out with a three-electrode system, where the NPG was used as the working electrode and a mixed solution of $0.1 \mathrm{M} \mathrm{Mn}\left(\mathrm{CH}_{3} \mathrm{COO}\right)_{2}$ and $0.1 \mathrm{M} \mathrm{Na}_{2} \mathrm{SO}_{4}$ as electrolyte. The loading of $\mathrm{MnO}_{2}$ can be easily adjusted by changing the deposition time and constant current density. The charge-discharge storage capacity of the hybrid NPG@MnO2 electrode was measured in $1 \mathrm{M}$ aqueous $\mathrm{Na}_{2} \mathrm{SO}_{4}$ solution. When the current density was increased from 0.5 to 8 $\mathrm{mA} \cdot \mathrm{cm}^{-2}$, the areal capacitance decreased from 45 to $37.2 \mathrm{mF} \cdot \mathrm{cm}^{-2}$, corresponding to a decrease in mass capacitance from 839.4 to $690.1 \mathrm{~F} \cdot \mathrm{g}^{-1}$, which shows a better capacitive property than other reported $\mathrm{MnO}_{2}$-based nanostructures like $\mathrm{MnO}_{2} /$ carbon nanotube $\left(\sim 385.4 \mathrm{~F} \cdot \mathrm{g}^{-1}\right)[17]$, $\mathrm{MnO}_{2}$ /polyaniline $\left(\sim 415 \mathrm{~F} \cdot \mathrm{g}^{-1}\right)$ [18] and $\mathrm{MnO}_{2}$ /nanotube/PEDOT-poly(styrenesulphonate) ternary composites $\left(\sim 427 \mathrm{~F} \cdot \mathrm{g}^{-1}\right)[19]$. 


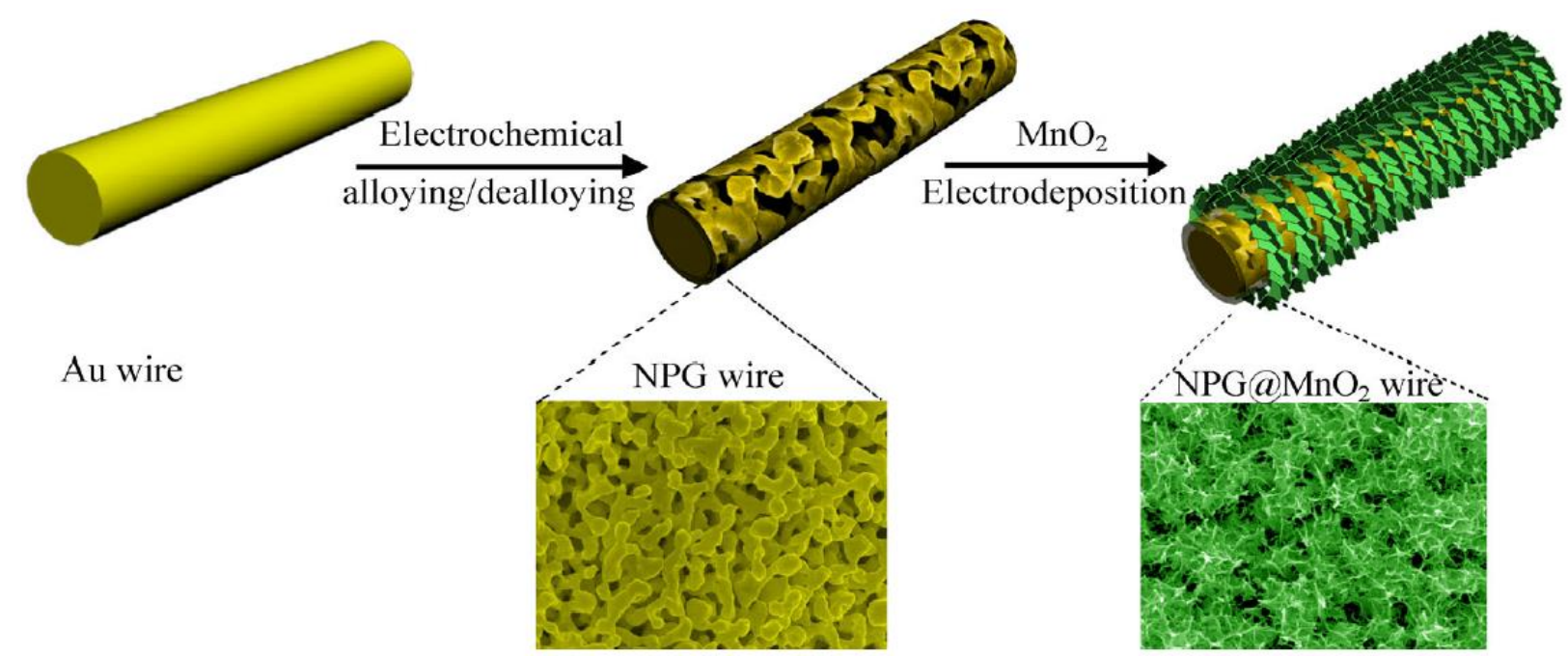

Fig. 2 Schematic illustration of the synthesis and morphology of the NPG@MnO2 electrode[16].

Among many types of metal oxides,nickel hydroxide $\left(\mathrm{Ni}(\mathrm{OH})_{2}\right)$ has been widely researched due to its potential for high energy density[20]. Sun-I Kim et al.[21]have developed an outstanding supercapacitor with high capacitance, high energy density, and semipermanent lifetime using the $\mathrm{Ni}(\mathrm{OH})_{2} / \mathrm{NPG}$ electrode. By synthesizing a hierarchical structure of $\mathrm{Ni}(\mathrm{OH})_{2}$ on a porous 3D-current collector, the capacitance per gram and per volume has been maximized to reach $3168 \mathrm{~F} \cdot \mathrm{g}^{-1}$ and $2223 \mathrm{~F} \cdot \mathrm{cm}^{-3}$, respectively. The low resistance metal-semiconductor contact effectively decreased the loss of capacitance by lowering the dead volume at the interface between the $\mathrm{Ni}(\mathrm{OH})_{2}$ and NPG. By maintaining the volume of inner pores, the effects of the nanochannels were enhanced in the unique structure of the porous electrode, which dramatically improved the capacitive properties and cycle stability.

In Xingyou Lang's study[22], three-dimensional bicontinuous NPG/PANI composite electrodes have been fabricated by electrochemical polymerization. The freestanding and flexible composite films have been demonstrated a volumetric capacitance up to $1500 \mathrm{~F} \mathrm{~cm}^{-3}$ at the current density of $\sim 1$ $\mathrm{A} \mathrm{cm}^{-3}, 10$ times higher than that of graphene/PANI composites $\left(\sim 150 \mathrm{~F} \cdot \mathrm{cm}^{-3}\right)$, which make this composite a promising electrode material for high-performance aqueous and all-solid-state supercapacitors with ultrahigh power density, high energy density. The good capacitive behaviors of the NPG/PANI SC devices arise from the ion and electron transports enhanced by unique bicontinuous nanostructure, where the nanoporosity facilitates the fast ion diffusion, and provides the large PANI/electrolyte interface to ensure the sufficient redox reaction of PANI during charge/discharge processes. The three-dimensional interconnected $\mathrm{Au}$ network with ultrahigh electrical conductivity harnesses the electron transport by remarkably decreasing the internal resistance of assembled devices.

\section{Conclusion and prospect}

In conclude, NPG with quasi-periodic nanoporous channels, a large internal surface and excellent conductivity shows promising application prospect in supercapacitor electrodes. There has been some progress in nanoporous gold-based supercapacitor such as combining different transition metal oxides and conductive polymers with NPG for optimized capacitive property. More work could be done to improve NPG electrode performance by controlling the nanoporosity due to the convenient access to pore size control. Flexible NPG can also be optimized for further application in wearable device with appropriate electrolyte.

\section{References}

[1] A.S. Aricò, P. Bruce, B. Scrosati, J. Tarascon, W. van Schalkwijk, Nat. Mater. 4(2005) 366377. 
[2] N.-S. Choi, Z. H. Chen, S. A. Freunberger, X. L. Ji, Y. K. Sun, K. Amine, G. Yushin, L. F. Nazar, J. Cho and P. G. Bruce, Angew. Chem., Int. Ed., 2012, 51, 9994-10024.

[3] P. Simon, Y. Gogotsi, Nat. Mater. 7 (2008) 845.

[4] Yonggang Wang, Yanfang Song and Yongyao Xia, Chem. Soc. Rev., 2016, 45, 5925-5950.

[5] X Yu B T, Lee J M. Graphene for supercapacitor applications[J]. Journal of Materials Chemistry A, 2013, 1(47):14814-14843.

[6] Faraji S, Ani F N. The development supercapacitor from activated carbon by electroless plating-A review[J]. Renewable \& Sustainable Energy Reviews, 2015, 42:823-834.

[7] X.Y. Lang, H.T. Yuan, Y. Iwasab and M.W. Chen, Scripta Materialia 64 (2011) 923-926.

[8] Wittstock A, Bienera J, Bäumerb M. Introduction to nanoporous gold[J]. 2011:1-10.

[9] Juergen Biener, Andrea M. Hodge, Alex V. Hamza, Luke M. Hsiung, and Joe H. Satcher, Jr. Journal of Applied Physics 97, 024301 (2005).

[10]G. P. Wang, L. Zhang and J. J. Zhang, Chem. Soc. Rev., 2012,41, 797-828.

[11]X.Y. Lang, H.T. Yuan, Y. Iwasa and M.W. Chen. Scripta Materialia 64 (2011) 923-926.

[12]Conway B E. Electrochemical supercapacitors[M]. New York: Kluwer Academic/Plenum Publishers, 1999:(a)259-289;(b)609.

[13]S.W. Lee, J. Kim, S. Chen, P.T. Hammond, Y. Shao-Horn, ACS Nano 7 (2010) 3889.

[14]Kuo-chan L, Mare A, Anderson. Porous nickel oxide/nickel films for electrochemical capacitors[J]. J electrochem Soc,1996,143(1):124-129.

[15]Chuan L, James A, Branko N. Povov, Characterization of sol-gel derived cobalt xergels as electrochemical capacitor[J]. J Electrochem Soc,1998,145(12):4097-4101.

[16]Henghui Xu, Xianluo Hu, Yongming Sun, Huiling Yang, Xiaoxiao Liu, and Yunhui Huang. Nano Research 2015, 8(4): 1148-1158.

[17]Zhou, Y. K., He, B. L., Zhang, F. B. \& Li, H. L. Hydrous manganese oxide/carbon nanotube composite electrodes for electrochemical capacitors. J. Solid State Electrochem. 8, 482-487 (2004).

[18]Chen, L. et al. Synthesis and pseudocapactive studies of composite films of polyaniline and manganese oxide nanoparticles. J. Power Sources 195,3742-3747 (2010).

[19]Hou, Y., Cheng, Y. W., Hobson, T. \& Liu J. Design and synthesis of hierarchical $\mathrm{MnO}_{2}$ nanospheres/carbon nanotubes/conducting polymer ternary composite for high performance electrochemical electrodes. Nano Lett. 10,2727-2733 (2010).

[20]H.B.Li,et al.,Amorphous nickel hydroxide nanospheres with ultrahigh capacitance and energy density as electrochemical pseudocapacitor materials,Nat.Commun. (2013)1894.

[21]Sun-I Kim , Sung-WookKim , KyoungokJung , Jin-BaekKim , Ji-HyunJang. Nano Energy24 (2016) 17-24.

[22] Xingyou Lang, Ling Zhang, Takeshi Fujita, Yi Ding, Mingwei Chen. Journal of Power Sources 197 (2012) 325- 329. 